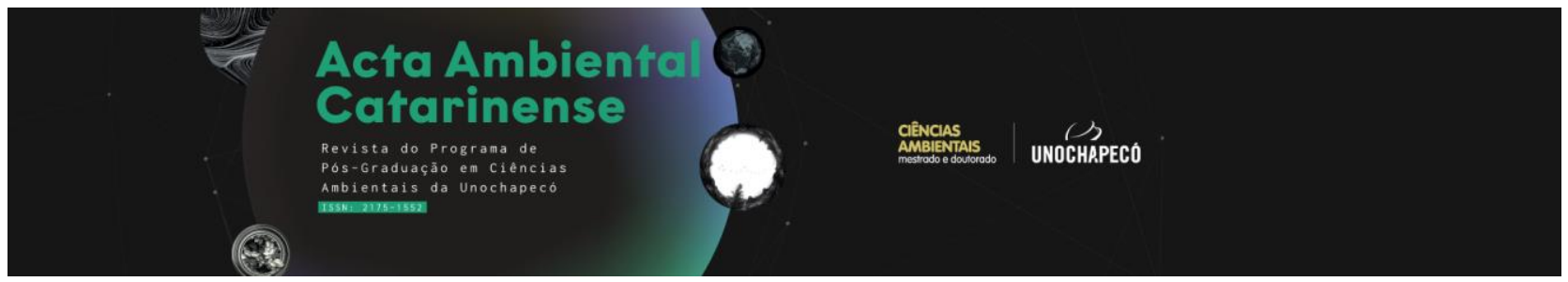

\title{
MUDANÇAS NO CLIMA E A DISTRIBUIÇÃO POTENCIAL FUTURA DE MIMOSA SCABRELLA BENTH
}

\author{
Mariéle Alves Ferrer da Silva ${ }^{1}$ \\ Pedro Higuchi ${ }^{2}$ \\ Ana Carolina da Silva ${ }^{3}$
}

\begin{abstract}
Resumo
O aquecimento global representa atualmente uma importante ameaça à biodiversidade. Desta forma, objetivou-se estimar como a distribuição potencial de Mimosa scabrella Benth. será impactada por mudanças no clima. Para a ocorrência geográfica da espécie, foram consideradas 19 variáveis bioclimáticas e a altitude. O nicho bioclimático foi modelado por intermédio do algoritmo de Máxima Entropia (Maxent). A qualidade dos ajustes foi verificada por meio dos valores de TSS. Foram realizadas projeções para o ano de 2070, utilizando-se o consenso dos ajustes com TSS > 0,85, considerando um cenário otimista (RCP4.5) e um pessimista (RCP8.5), conforme o quinto relatório do IPCC. M. scabrella apresenta distribuição fitogeográfica de caráter subtropical, sendo influenciada principalmente pela quantidade de chuva no verão e isotermalidade. Nos cenários mais e menos otimistas estima-se que haverá uma redução de, respectivamente, 21,7 e 48,9\% em sua área de ocorrência potencial. Em se tratando de áreas estratégicas para a conservação da espécie, se destacaram as áreas planálticas e serranas na região Sul e Sudeste.
\end{abstract}

Palavras-chave: Bracatinga; Conservação; Nicho climático.

\begin{abstract}
At present, global warming represents a significant threat to biodiversity. Thus, this study aimed to estimate how the potential distribution of Mimosa scabrella Benth. will be impacted by climate changes. For the geographical occurrence of the species, we considered 19 bioclimatic variables and altitude. We modeled the bioclimatic niche the maximum entropy algorithm (Maxent). We checked the quality of the adjustments through TSS values. Consensual projections for 2070 were obtained using only the adjustments with TSS $>0.85$, considering an optimistic scenario (RCP4.5) and a pessimistic one (RCP8.5), according to the fifth report of the IPCC. M. scabrella presents a

\footnotetext{
${ }^{1}$ Universidade do Estado de Santa Catarina; Departamento de Engenharia Florestal, CAV/UDESC, Av. Luiz de Camões, 2090, B. Conta Dinheiro, Cep. 88520-000, Lages, SC, Brasil.

${ }^{2}$ Universidade do Estado de Santa Catarina; Departamento de Engenharia Florestal, CAV/UDESC, Av. Luiz de Camões, 2090, B. Conta Dinheiro, Cep. 88520-000, Lages, SC, Brasil. Email: higuchip@ gmail.com

${ }^{3}$ Universidade do Estado de Santa Catarina; Departamento de Engenharia Florestal, CAV/UDESC, Av. Luiz de Camões, 2090, B. Conta Dinheiro, Cep. 88520-000, Lages, SC, Brasil. 
subtropical geographic distribution, being affected mostly by rains during summers and isothermality. In the more and less optimistic scenarios, it is estimated that there will be a reduction of 21.7 and $48.9 \%$ respectively in their potential area of occurrence. Regarding the strategic areas for the conservation of the species, the highland and plateau areas in the South and Southeast regions were particularly noteworthy.

Keywords: Bracatinga; Conservation; Climate niche

\section{INTRODUÇÃ̃O}

As mudanças climáticas são umas das maiores preocupações mundiais da atualidade (PECL et al., 2017). Estima-se que cerca de uma em cada seis espécies poderá se extinguir em decorrência do aquecimento global, sendo que a América do Sul poderá ser uma das regiões do planeta mais fortemente impactadas (URBAN, 2015). Além disso, as alterações climáticas poderão resultar na migração e adaptação de espécies, tanto animais quanto vegetais, para regiões que apresentem condições semelhantes de onde ocorrem naturalmente (CORLETT e WESTCOTT, 2007).

O clima desempenha um papel de grande relevância para a adaptação e o crescimento das espécies vegetais, portanto, se torna indispensável a definição de ações que busquem prevenir ou mitigar os impactos causados à biodiversidade, ocasionados pelas mudanças climáticas (HOFFMAN e SGRÒ, 2011; TOLEDO et al., 2011). Neste sentido, compreender as interações entre as mudanças climáticas, a distribuição e a dinâmica da vegetação está se tornando um dos maiores desafios para a conservação dos ecossistemas naturais (SVENNING e SANDEL, 2013). Sendo assim, estudos relacionados à distribuição geográfica de espécie florestais são fundamentais para prever os impactos das mudanças climáticas futuras sobre a vegetação (HIGUCHI et al., 2013).

Os modelos de nichos climáticos têm sido frequentemente utilizados para prever a distribuição de espécies, dando suporte às estratégias de recuperação e conservação da vegetação (KEENAN et al., 2011). Esses modelos permitem avaliar as condições ambientais responsáveis pelas distribuições das espécies e os resultados obtidos podem ser extrapolados considerando o clima futuro, chegando a uma predição de onde estas condições poderão ocorrer (ELITH et al., 2011). Ou seja, esses resultados podem indicar se o local será adequado ou não para o desenvolvimento da espécie (GIANNINI et al., 2012).

Segundo Bertoldo et al. (2014), a Floresta Ombrófila Mista (FOM) pode ser considerada uma das fitofisionomias mais sensíveis às variações climáticas. A distribuição de muitas espécies encontradas nessa formação florestal apresenta alta correlação com a temperatura, mostrando que a sensibilidade a essas mudanças pode afetar os seus crescimentos (KANIESKI et al., 2012). A FOM apresenta sua maior área de distribuição geográfica associada aos locais de elevada altitude e com temperaturas médias anuais relativamente baixas, ocorrendo de forma associada à vegetação campestre (HIGUCHI et al., 2012).

Considerando este contexto, o presente trabalho buscou estudar a distribuição potencial presente e futura de Mimosa scabrella Benth. (bracatinga), uma espécie associada à FOM, com destaque para os estados do RS, SC, PR e SP, entre as latitudes $21^{\circ} 30^{\prime} \mathrm{S}$ e $29^{\circ} 40^{\circ} \mathrm{S}$ e longitudes $48^{\circ}$ $30^{\prime} \mathrm{W}$ e $53^{\circ} 50^{\prime} \mathrm{W}$ (SOBIERAJSKI, 2004; AMARAL et al., 2015), com clima subtropical úmido e subtropical de altitude, sem períodos de seca prolongados. Como é uma espécie pioneira e resistente a geadas, desenvolve-se bem a céu aberto, ou em clareiras dentro da floresta (FERREIRA et al., 2019; MARTINS et al., 2019; PARIZOTTO et al., 2019). Também ocorre em florestas 
secundárias, caracterizando-se como espécie pioneira (STEENBOCK et al., 2011). Por possuir rápido crescimento e elevada produtividade em altas densidades, as populações naturais de bracatinga vêm sendo manejadas por agricultores de diferentes regiões do Sul do Brasil (STEENBOCK et al., 2011), principalmente para a produção de lenha e carvão (FRIEDERICHS et al., 2015). Também destacam-se outros usos potenciais, tais como em plantios para recuperação de áreas degradadas (FERREIRA et al., 2019), fabricação de painéis de madeira (NAPOLI et al, 2013), moirões (PAES et al., 2015) e produção de mel de melato (AZEVEDO et al., 2017).

Considerando a importância da bracatinga como espécie colonizadora, assim como a importância econômica da espécie, objetivou-se com o presente estudo conhecer a sua distribuição geográfica potencial, a fim de predizer o impacto das mudanças climáticas futuras. Com isso, espera-se contribuir para a definição de áreas estratégicas para a conservação da espécie, que possam potencialmente servir como refúgios climáticos.

\section{Material e Métodos}

As coordenadas geográficas de $M$. scabrella foram extraídas do Banco de Dados do LABDENDRO/UDESC, da literatura científica (FORMENTO et al., 2004; SOBIERAJSKI, 2004; MORIM， 2006; KOZERA et al., 2006; GARCIA, 2007; HERRERA et al., 2008; LIEBSCH et al., 2009; MOREIRA, 2009; SAWCZUK, 2009; CORDEIRO, 2010; RAMOS et al., 2011; FERREIRA et al., 2012; SANTOS et al., 2012; HIGUCHI et al., 2013) e do banco de dados da Rede SpeciesLink (HERBÁRIO ALEXANDRE LEAL COSTA et al., 2019)

Para explicar a ocorrência da espécie, foram empregadas 19 variáveis bioclimáticas e a altitude, obtidas do WordClim (HIJMANS et al., 2005), na resolução de 2,5 minutos ( $~ 5$ $\mathrm{Km})$ : bio 1 (temperatura média anual), bio 2 (média da amplitude térmica diária), bio 3 (isotermalidade), bio 4 (sazonalidade térmica), bio 5 (temperatura máxima no mês mais quente), bio 6 (temperatura mínima no mês mais frio), bio 7 (amplitude térmica anual), bio 8 (temperatura média no trimestre mais úmido), bio 9 (temperatura média no trimestre mais seco), bio 10 (temperatura média no trimestre mais quente), bio 11 (temperatura média no trimestre mais frio), bio 12 (precipitação anual), bio 13 (precipitação no mês mais úmido), bio 14 (precipitação no mês mais seco), bio 15 (sazonalidade da precipitação), bio 16 (precipitação no trimestre mais úmido), bio 17 (precipitação no trimestre mais seco), bio 18 (precipitação no trimestre mais quente) e bio 19 (precipitação no trimestre mais frio). Estas variáveis indicam tendências gerais, valores extremos e amplitudes de temperatura e precipitação.

Baseado no quinto relatório do IPCC, foram considerados dois cenários de mudanças do clima para o ano de 2070, sendo um mais otimista (RCP4.5) e outro mais pessimista (RCP8.5). No RCP4.5, a civilização moderna adotará medidas para reduzir a emissão de gases causadores do efeito estufa. No RCP8.5, espera-se que nada será feito para diminuir o efeito estufa. Como Modelo de Circulação Atmosférica será empregado o HADGEM2-ES, que faz parte do Coupled Model Intercorporation Project Phase 5 (CMIP5). Os bioclimas para o RCP4.5 e RCP8.5 foram extraídos do WordClim (HIJMANS et al., 2005).

Como sugerido por Hijmans e Elith (2017), as coordenadas geográficas foram verificadas quanto à existência de ocorrências duvidosas, sendo estas removidas das análises subsequentes. Além disso, em função de possíveis viés relacionados à ocorrência de pontos espacialmente auto-correlacionados, as coordenadas geográficas foram espacialmente filtradas (BORIA et al., 2014), considerando-se apenas uma ocorrência a cada $2,5 \times 2,5 \mathrm{~km}$. Para remover as varáveis explicativas multicolineares, foram 
calculados os Valores de Fator de Inflação da Variância (VIF), com remoção daquelas com VIF > 10 (KHANUM et al., 2013).

A distribuição geográfica da espécie foi modelada por meio do algoritmo de Máxima Entropia (Maxent) (PHILLIPS et al., 2017). Pelo fato dos dados de ocorrência geográfica representarem apenas informações relacionadas à presença da espécie, foi realizada a geração de forma completamente aleatória de um total de 10.000 pseudoausências, distribuídas em um raio de até 500 $\mathrm{km}$, no entorno de cada registro da espécie (BARBET-MASSIN et al., 2012).

A acurácia da modelagem foi verificada por meio dos valores de TSS (True Skill Statistics), que varia de -1 a 1, cujo valor máximo indica um ajuste perfeito (SHABANI et al., 2018). Os valores de TSS foram determinados a partir do procedimento de calibração (70\% dos dados) e teste (30\% restante dos dados) (SIQUEIRA et al., 2019), que foi repetido por cinco vezes.

A relação entre a probabilidade de ocorrência da espécie e as variáveis de maior contribuição no processo de modelagem foi analisada por meio de curvas de respostas. A projeção contemporânea e futura de $M$. scabrella foi realizada a partir de um consenso dos ajustes considerados de boa acurácia (TSS $>$ 0,40) (SIQUEIRA et al., 2019). Projeções binárias, contemporâneas e futuras, foram criadas a partir de uma probabilidade de ocorrência da espécie superior a 50\%, que, posteriormente, foram sobrepostas, a fim de se quantificar o impacto dos diferentes cenários de mudanças climáticas. As análises foram realizadas por meio do R (R CORE TEAM, 2019), junto com os pacotes usdm (NAIMI, 2019), dismo (HIJMANS et al., 2019), raster (HIJMANS, 2019) e biomod2 (THUILLER et al., 2019).

\section{Resultados}

Após remoção das variáveis altamente correlacionadas entre si, além da altitude, oito variáveis bioclimáticas (bio2, bio3, bio8, bio
13, bio14, bio15, bio18 e bio19) foram utilizadas para a modelagem da distribuição de M. scabrella (Tabela 1, Figura 1). Como base nos registros geográficos, nota-se que $M$. scabrella apresenta uma ocorrência restrita ao Sul e Sudeste do Brasil (Figura 1).

Tabela 1. Variáveis usadas como preditoras da distribuição geográfica de Mimosa scabrella, após remoção de colinearidade.

\begin{tabular}{lll}
\hline Sigla & Variável & VIF \\
\hline Alt & Altitude & 9,00 \\
bio2 & Amplitude diurna média & 2,66 \\
bio3 & Isotermalidade & 2,45 \\
bio8 & Temperatura média do trimestre & 6,99 \\
& mais úmido & \\
bio13 & Precipitação do mês mais chuvoso & 4,02 \\
bio14 & Precipitação do mês mais seco & 4,26 \\
bio15 & Sazonalidade da precipitação & 2,68 \\
bio18 & Precipitação do trimestre mais & 3,05 \\
& quente & \\
bio19 & Precipitação do trimestre mais frio & 3,71 \\
\hline
\end{tabular}

O valor médio de TSS para os ajustes foi de $0,71(\min =0,619 ; \max =0,748)$, sendo, por tanto, considerados como de boa acurácia. A precipitação no trimestre mais quente e a isotermalidade foram as variáveis de maior importância para explicar a distribuição geográfica de $M$. scabrella. Ao se analisar as curvas de probabilidade de ocorrência da espécie em função das variáveis mais explicativas, observa-se que $M$. scabrella teve baixa probabilidade de ocorrência em locais com verões mais secos e de maior isotermalidade (Figura 3). Assim, de forma geral, os modelos indicam que a espécie ocorre preferencialmente em regiões subtropicais com verões úmidos.

A predição espacial para o Sul do Brasil (Figura 4) indica que as áreas com elevada probabilidade de ocorrência de $M$. scabrella coincidem, em grande extensão, com a de Floresta Ombrófila Mista. Observase mudanças nas áreas de ocorrência potencial diante os diferentes cenários de mudanças climáticas. As áreas de ocorrência potencial perdidas concentram-se, predominantemente, nas regiões periféricas, associadas aos locais 
de menor altitude. As áreas que passarão a ter adequabilidade climática foram pouco expressivas, ocorrendo de forma esparsa na região. No melhor cenário de mudanças climáticas (RCP 4.5), observa-se uma redução na área de ocorrência potencial de $21,7 \%$; e no pior cenário (RCP 8.5), uma redução de área de $48,9 \%$.


Figura 1. Distribuição de Mimosa scabrella e as variáveis bioclimáticas preditivas utilizadas na modelagem do nicho climático (alt - altitude, bio2 - amplitude diurna média, bio3 isotermalidade, bio8 - temperatura média do trimestre mais úmido, bio13 - precipitação do mês mais chuvoso, bio14 - precipitação do mês mais seco, bio15 - sazonalidade da precipitação, bio18 - precipitação do trimestre mais quente e bio19 - precipitação do trimestre mais frio). 



Figura 2. Probabilidade de ocorrência de Mimosa scabrella, ao longo dos gradientes bioclimáticos de maior significância (precipitação no trimestre mais quente e isotermalidade).

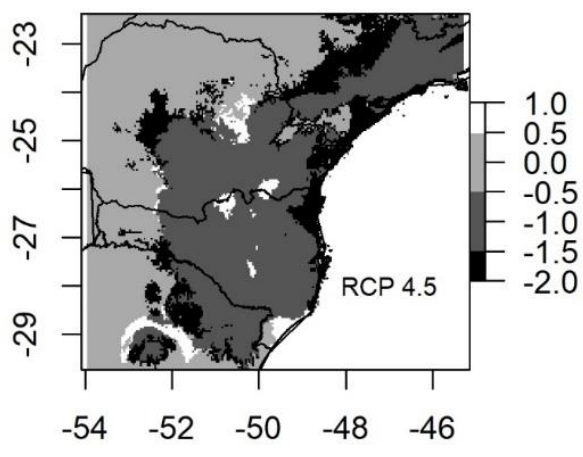

a

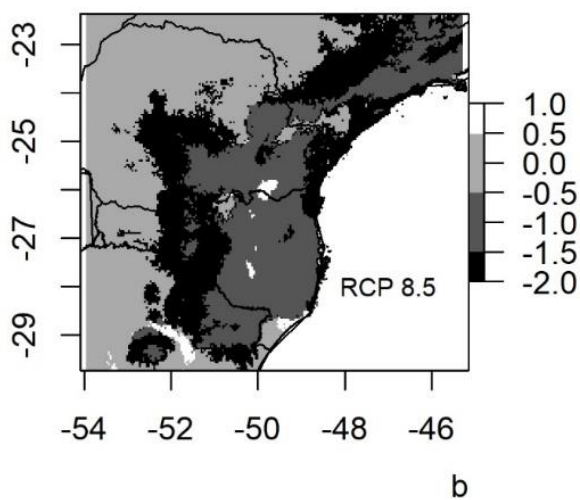

Figura 3. Impacto de mudanças climáticas sobre a área de ocorrência potencial de Mimosa scabrella, conforme cenários menos (RCP 4.5) e mais pessimista (RCP 8.5), previsto pelo modelo de circulação atmosférica HadGEM2-AO, para o ano de 2070. Cinza escuro = Área estável, com elevada adequabilidade climática tanto no presente quanto no futuro; Cinza claro = Área estável, com baixa adequabilidade climática tanto no presente quanto no futuro; Preto $=$ Área contemporânea não-estável (com elevada adequabilidade climática apenas no presente); Branco: Área futura não-estável (com elevada adequabilidade climática apenas no futuro). 


\section{DISCUSSÃO}

Os resultados demonstraram que $M$. scabrella ocorre em domínio predominantemente Atlântico, com maior concentração nas regiões Sul e Sudeste do Brasil e, segundo Amaral et al. (2015), com uma relação de maior associação com à Floresta Ombrófila Mista. O clima demonstrou ser um importante elemento ecológico na determinação da área de ocorrência da espécie, como ficou demostrado a partir da relevância das variáveis relacionadas à precipitação pluviométrica e à amplitude de temperatura. De forma geral, além de ocorrer preferencialmente no Sul e Sudeste do Brasil, M. scabrella apresenta elevada probabilidade de ocorrência em regiões de verões úmidos, denotando um clima tipicamente temperado úmido, que se enquadra na classificação $\mathrm{Cfb}$ e Cfa de Koppen. A partir de estudos sobre a filogenia desta espécie (MORALES et al., 2014), é possível inferir que seu nicho bioclimático pode estar relacionado com sua história evolutiva. Mimosa é um dos gêneros de maior riqueza dentro da família Leguminosa, com mais do que 500 espécies, sendo que a região subtropical da América do Sul (Paraguai, Argentina, Uruguai e Sul do Brasil) pode ser considerada como um importante centro de diversidade endemismo (SIMON et al., 2011). Como destacado por Morales et al. (2014), a origem das espécies deste grupo ainda é desconhecida e está relacionada com um ancestral em comum provavelmente já extinto.

Enquanto as áreas de menor altitude serão mais impactadas diante dos cenários de mudanças climáticas, aquelas situadas em maiores cotas altitudinais tenderão a apresentar maior estabilidade, o que está de acordo com estudos recentes que avaliaram a influência do clima sobre a ocorrência de Araucaria angustifolia no Sul do Brasil (BERGAMIN et al. 2019; MARCHIORO et al., 2019; WILSON et al., 2019), reforçando a importância da conservação destas áreas como potenciais refúgios climáticos. Destaca-se que como observado em eventos pretéritos de mudanças do clima, como aqueles que ocorreram no Quaternário, a alteração das áreas de ocorrências geográficas de espécies ao longo de gradientes altitudinais e latitudinais é uma resposta comum (CHEN et al., 2011). No entanto, estudos têm demostrado que o processo de mudanças climáticas tem ocorrido com elevada rapidez (CHEN et al., 2011; WEBBER; SCOTT, 2012), o que torna uma incógnita a capacidade de respostas das espécies, principalmente, quando contextualizada junto com outras influências de natureza antrópica, como a alteração do uso solo (CUNZE et al., 2013).

Conclui-se que $M$. scabrella tem um nicho bioclimático caracterizado por um clima subtropical e com verões úmidos. Diante dos cenários de mudanças climáticas avaliadas, estima-se que a espécie apresentará redução da área de adequabilidade climática para a sua ocorrência natural. As áreas planálticas e serranas na porção subtropical da Floresta Atlântica têm $o$ potencial de funcionarem como refúgios climáticos, portanto são estratégicas para conservação.

\section{Agradecimentos}

À FAPESC, pela concessão de bolsa à primeira autora e pelo apoio por meio do PAP/UDESC. Ao CNPq, pela bolsa de produtividade ao segundo e terceiro autores.

\section{REFERÊNCIAS}

AMARAL GM, FONTANA C, GASPER AL, FREITAS DS, SEVEGNANI L. Aspectos da distribuição de Mimosoideae (Fabaceae) arbóreas no planalto de Santa Catarina, sul do Brasil. Neotropical Biology and Conservation, São Leopoldo, v. 10, n. 2, p. 74-84, 2015.

AZEVEDO MS et al. Proteome comparison for discrimination between honeydew and

Vol. 18, N. 01 (2021) 
floral honeys from botanical species Mimosa scabrella Bentham by principal component analysis. Journal of the Science of Food and Agriculture, Hoboken, v. 97, n. 13, p. 45154519, 2017.

BARBET-MASSIN M, JIGUET F, ALBERT $\mathrm{CH}$, THUILLER W. Selecting pseudoabsences for species distribution models: how, where and how many? Methods Ecol Evol, Hoboken, v.3. p. 327-338, 2012

BERGAMIN RS, DEBASTIANI V, JONER DC, LEMES P, GUIMARÃES T, LOYOLA RD, MÜLLER SC. Loss of suitable climatic areas for Araucaria forests over time. Plant Ecology \& Diversity, Abingdon-on-Thames, v. 12, p. 115-126, 2019.

BERTOLDO É, PAISANI JC, OLIVEIRA, PE. Registro de Floresta Ombrófila Mista nas regiões sudoeste e sul do Estado do Paraná, Brasil, durante o Pleistoceno/Holoceno. Hoehnea. São Paulo, v.41, n.1, p.1-8, 2014.

BORIA RA, OLSON LE, GOODMAN SM, ANDERSON RP. Spatial filtering to reduce sampling bias can improve the performance of ecological niche models. Ecological Modelling, Amsterdã, v. 275, p. 73-77, 2014.

CHEN I-C, HILL JK, OHLEMÜLLER R, ROY DB, THOMAS CD. Rapid range shifts of species associated with high levels of climate warming. Science, Washington, D.C., v. 333, n. 6045, p. 1024-1026, 2011.

CORDEIRO J. Compartimentação pedológico-ambiental e sua influência sobre a florística e estrutura de um remanescente de Floresta Ombrófila Mista na região centro sul do Paraná. 2010. Tese (Doutorado em Ciências Florestais) - UFPR, Curitiba, 2010.

CORLETT, RT.; WESTCOTT, DA. Will plant movements keep up with climate change? Trends in ecology \& evolution, Amsterdan, v. 28, n. 8, p. 482-488, 2013.

CUNZE S, HEYDEL F, TACKENBERG O. Are plant species able to keep pace with the rapidly changing climate? PloS one, San Francisco, v. 8, n. 7, p. e67909, 2013.

ELITH J, PHILLIPS SJ, HASTIE T, DUDÍK M, CHEE YE, YATES CJ. A statistical explanation of MaxEnt for ecologists. Diversity and distributions, Hoboken, v. 17, n. 1, p. 43-57, 2011.

FERREIRA PI, PALUDO GF, CHAVES CL, BORTOLUZZI RLC, MANTOVANI A. Florística e fitossociologia arbórea de remanescentes florestais em uma fazenda produtora de Pinus spp. Floresta, Curitiba, v. 42, n. 4, p. 783-794, 2012.

FERREIRA, PI et al. Mimosa scabrella Benth. as Facilitator of Forest Successional Advance in the South of Brazil. Floresta e Ambiente, Curitiba, v. 26, n. 4, 2019.

FORMENTO S, SCHORN LA, BLEY RRA. Dinâmica estrutural arbórea de uma Floresta Ombrófila Mista em Campo Belo do Sul, SC. Cerne, Lavras, v. 10, n. 2, p. 196-212, 2004.

GARCIA OP. Estrutura e composição do estrato arbóreo em diferentes trechos da Reserva Biológica Municipal Santa 
Cândida, Juiz de Fora-MG. 2007. Dissertação (Mestrado em Ecologia) - UFJF. Juiz de Fora, 2007.

FRIEDERICHS G, BRAND MA, CARVALHO AF, KÜSTER LC. Qualidade da madeira e do carvão vegetal de bracatinga (Mimosa scabrella Benth.). Brazilian Journal of Wood Science, Pelotas, v. 6, n. 2, 2015.

GIANNINI TC, SIQUEIRA MF, ACOSTA AL, BARRETO FCC, SARAIVA AM, ALVES-DOS-SANTOS I. Desafios atuais da modelagem preditiva de distribuição de espécies. Rodriguésia, Rio de Janeiro, v. 63, n. 3, p. 733-749, 2012.

HERBÁRIO ALEXANDRE LEAL COSTA (ALCB), ARIZONA STATE UNIVERSITY VASCULAR PLANT HERBARIUM (ASUPlants), XILOTECA CALVINO MAINIERI (BCTw), HERBÁRIO DA UFMG FANERÓGAMAS, ALGAS E FUNGOS (BHCB), BRAZILIAN LABORATORY OF AGROSTOLOGY (BLA), HERBÁRIO IRINA DELANOVA GEMTCHÚJNICOV (BOTU), HERBÁRIO DA EMBRAPA RECURSOS GENÉTICOS E BIOTECNOLOGIA (CEN), HERBÁRIO DO CENTRO DE PESQUISAS DO CACAU (CEPEC), HERBÁRIO LEOPOLDO KRIEGER (CESJ), HERBÁRIO DA FUNDAÇÃO UNIVERSIDADE FEDERAL DE MATO GROSSO DO SUL (CGMS), HERBÁRIO CPAP DA EMBRAPA PANTANAL (CPAP), HERBÁRIO PE. DR. RAULINO REITZ (CRI), HERBÁRIO DE CURITIBANOS (CTBS), HERBÁRIO DA UNIVERSIDADE TECNOLÓGICA FEDERAL DO PARANÁ - DOIS VIZINHOS (DVPR), HERBÁRIO PRISCO BEZERRA (EAC), HERBÁRIO ESCOLA DE FLORESTAS CURITIBA (EFC),
HERBÁRIO DA ESCOLA SUPERIOR DE AGRICULTURA LUIZ DE QUEIROZ (ESA), HERBÁRIO ESAL (ESAL), EVALDO BUTTURA (EVB), FIELD MUSEUM OF NATURAL HISTORY (BOTANY) SEED PLANT COLLECTION (F), HERBÁRIO DO DEPARTAMENTO DE BOTÂNICA DA UNIVERSIDADE FEDERAL DE SANTA CATARINA (FLOR), FOTOTECA PAULO SCHWIRKOWSKI (FPS), HERBÁRIO DA UNIVERSIDADE ESTADUAL DE LONDRINA (FUEL), HERBÁRIO DR. ROBERTO MIGUEL KLEIN (FURB), GENEVA HERBARIA CATALOGUE WITH SPECIES BRAZIL (G), HERBÁRIO ALARICH RUDOLF HOLGER SCHULTZ (HAS), HERBÁRIO DA UNIVERSIDADE TECNOLÓGICA FEDERAL DO PARANÁ CAMPUS CAMPO MOURÃO (HCF), HERBÁRIO DO DEPARTAMENTO DE CIÊNCIAS FLORESTAIS (HDCF), HERBÁRIO EZECHIAS PAULO HERINGER (HEPH), HERBÁRIO PADRE BALDUINO RAMBO (HPBR), HERBÁRIO DO JARDIM BOTÂNICO PLANTARUM (HPL), HERBÁRIO RIOCLARENSE (HRCB), HERBÁRIO DA UNIVERSIDADE ESTADUAL DO CENTRO-OESTE (HUCO), HERBÁRIO DA PONTIFÍCIA UNIVERSIDADE CATÓLICA DO PARANÁ (HUCP), HERBÁRIO DA UNIVERSIDADE DE CAXIAS DO SUL (HUCS), HERBARIO DA UNIVERSIDADE ESTADUAL DE FEIRA DE SANTANA (HUEFS), HERBÁRIO UEM (HUEM), HERBARIUM UBERLANDENSE (HUFU), HERBÁRIO PROF. JORGE PEDRO PEREIRA CARAUTA (HUNI), HERBÁRIO DO RECÔNCAVO DA BAHIA (HURB), HERBÁRIO DO VALE DO TAQUARI (HVAT), HERBÁRIO DO INSTITUTO AGRONÔMICO DE CAMPINAS (IAC), INSTITUTO AGRONÔMICO DO NORTE (IAN), HERBÁRIO DO INSTITUTO DE CIÊNCIAS NATURAIS (ICN), HERBÁRIO 
INPA (INPA), HERBÁRIO - IPA DÁRDANO DE ANDRADE LIMA (IPA), HERBÁRIO DO PARQUE DA CIÊNCIA NEWTON FREIRE MAIA (IRAI), HERBÁRIO JOINVILLEA (JOI), HERBÁRIO DE LAGES DA UNIVERSIDADE DO ESTADO DE SANTA CATARINA (LUSC), HERBÁRIO MACK (MACK), HERBÁRIO DO MUSEU BOTÂNICO MUNICIPAL (MBM), HERBÁRIO MELLO LEITÃO (MBMLHERBARIO), MISSOURI BOTANICAL GARDEN (MO), HERBÁRIO DO MUSEU DA PONTIFÍCIA UNIVERSIDADE CATÓLICA DO RIO GRANDE DO SUL (MPUC), NATURALIS BIODIVERSITY CENTER (NL) - BOTANY (NL-BOTANY), THE NEW YORK BOTANICAL GARDEN - SOUTH AMERICA RECORDS (NY), HERBÁRIO "PROFESSOR JOSÉ BADINI" (OUPR), HERBARIUM ANCHIETA (PACA-AGP), HERBÁRIO DO MUSEU NACIONAL (R), HERBÁRIO DO DEPARTAMENTO DE BOTÂNICA, INSTITUTO DE BIOLOGIA (RFA), HERBÁRIO RSPF (RSPF), SISTEMA DE INFORMAÇÃO DO PROGRAMA BIOTA/FAPESP (SINBIOTA), HERBÁRIO DA UNIVERSIDADE FEDERAL DE SANTA MARIA (SMDB), HERBÁRIO DO CENTRO DE CIÊNCIAS E TECNOLOGIAS PARA A SUSTENTABILIDADE (SORO), HERBÁRIO DO ESTADO "MARIA ENEYDA P. KAUFMANN FIDALGO" COLEÇÃO DE FANERÓGAMAS (SP), HERBÁRIO DA UNIVERSIDADE DE SÃO PAULO (SPF), XILOTECA DO INSTITUTO DE BIOCIÊNCIAS DA UNIVERSIDADE DE SÃO PAULO (SPFW), HERBÁRIO DOM BENTO JOSÉ PICKEL (SPSF), HERBÁRIO GRAZIELA BARROSO (TEPB), HERBÁRIO DA UNIVERSIDADE DE BRASÍLIA (UB), HERBÁRIO DA UNIVERSIDADE ESTADUAL DE CAMPINAS (UEC), HERBÁRIO UFP GERALDO MARIZ (UFP), HERBÁRIO DA
UNIVERSIDADE FEDERAL DO PARANÁ (UPCB), SMITHSONIAN DEPARTMENT OF BOTANY - SOUTH AMERICAN RECORDS (US), HERBÁRIO DA UNIVERSIDADE FEDERAL DE VIÇOSA (VIC), HERBÁRIO CENTRAL DA UNIVERSIDADE FEDERAL DO ESPÍRITO SANTO VIES (VIES) disponível na rede speciesLink (http://www.splink.org.br) em 09 de Junho de 2019

HIGUCHI P, SILVA AC, FERREIRA TS, SOUZA ST, GOMES JP, SILVA KM, SANTOS KF, LINKE C, PAULINO PS. Influência das variáveis ambientais sobre o padrão estrutural e florístico do componente arbóreo, em um fragmento de Floresta Ombrófila Mista Montana em Lages, SC. Ciência Florestal, Santa Maria, v. 22, n. 1, p. 79-90, 2012.

HIGUCHI P, SILVA AC, BUCKE JC, MANTOVANI A, BORTOLUZZI RLC, ZIGER AA. Influência do clima e de rotas migratórias de espécies arbóreas sobre o padrão fitogeográfico de florestas na região sul do Brasil. Ciência Florestal, Santa Maria, v. 23, n. 4, p. 539-553, 2013.

HIJMANS RJ, CAMERON SE, PARRA JL, JONES PG, JARVIS A. Very high resolution interpolated climate surfaces for global land areas. International Journal of Climatology, Hoboken, v. 25, n. 15, p. 19651978, 2005.

HIJMANS RJ. raster: Geographic Data Analysis and Modeling. R package version 2.3-40, 2019. 
HIJMANS RJ, ELITH J. Species distribution modeling with R. 2017.

HIJMANS RJ, PHILLIPS S, LEATHWICK J, ELITH J. dismo: Species Distribution Modeling. R package version 1.0-12, 2019.

HOFFMANN AA.; SGRÒ CM. Climate change and evolutionary adaptation. Nature, Londres, v. 470, n. 7335, p. 479-485, 2011.

KANIESKI MR, SANTOS TL, GRAF NETO J, SOUZA T, GALVÃO F, RODERJAN CV. Influência da precipitação e da temperatura no incremento diamétrico de espécies florestais aluviais em Araucária-PR. Floresta e Ambiente. Seropédica, v. 19, n. 1, p. 17-25, 2012.

KEENAN T, SERRA JM, LLORET F, NINYEROLA M, SABATE S. Predicting the future of forests in the Mediterranean under climate change, with niche- and process-based models: $\mathrm{CO}_{2}$ matters! Global Change Biology, Hoboken, v. 17, n. 1, p. 565-579, 2011.

KHANUM R, MUMTAZ AS, KUMAR S. Predicting impacts of climate change on medicinal asclepiads of Pakistan using Maxent modeling. Acta Oecologica, Amsterdã, v. 49, p. 23-31, 2013.

KOZERA C, DITTRICH VAO, SILVA SM. Composição florística da Floresta Ombrófila Mista Montana do Parque Municipal do Barigüi, Curitiba, PR. Floresta, Curitiba, v. 36, n. 1, p. 45-58, 2006.
LIEBSCH D, MIKICH SB, POSSETE RFS, RIBAS OS. Levantamento florístico e síndromes de dispersão em remanescentes de Floresta Ombrófila Mista na região centro-sul do estado do Paraná. Hoehnea, São Paulo, v. 36, n. 2, p. 233-248, 2009.

MARCHIORO CA, SANTOS KL, SIMINSKI A. Present and future of the critically endangered Araucaria angustifolia due to climate change and habitat loss. Forestry, Oxford, v. 0, p. 1-10, 2019.

MARTINS DAP et al. Espécies potenciais para recuperação de áreas degradadas a partir de levantamento florístico realizado no Planalto Catarinense. Revista de Ciências Agroveterinárias, Lages, v. 18, n. 5, p. 38-46, 2019.

MORALES M, WULFF AF, FORTUNATO RH, POGGIO L. Chromosome studies in southern species of Mimosa (Fabaceae, Mimosoideae) and their taxonomic and evolutionary inferences. Plant Systematics and Evolution, Nova York, v. 300, n. 5, p. 803-817, 2014.

MORIM MP. Leguminosae arbustivas e arbóreas da Floresta Atlântica do Parque Nacional do Itatiaia, Sudeste do Brasil: padrões de distribuição. Rodriguésia, Rio de Janeiro, v. 57, n. 1, p. 27-45, 2006.

NAIMI B. usdm: Uncertainty Analysis for Species Distribution Models. $\mathrm{R}$ package version 1.1-15, 2019.

NAPOLI LM et al. Propriedades físicas da madeira e de painéis aglomerados produzidos 
com misturas de espécies florestais. Floresta, Curitiba, v. 43, n. 3, p. 475-484, 2013.

PAES JB, MORESCHI JC, DE LELLES JG. Preservative treatment of Mimosa scabrella benth. and Eucalyptus viminalis lab. fence posts by dip-diffusion method. Cerne, Lavras, v. 7, n. 2, p. 065-080, 2015.

PARIZOTTO A et al. Florística e diversidade da regeneração natural em clareiras em Floresta Ombrófila Mista. Pesquisa Florestal Brasileira, Colombo, v. 39, n. 1, 2019.

PECL GT, ARAÚJO MB, BELL JD, BLANCHARD J, BONEBRAKE TC et al. Biodiversity redistribution under climate change: Impacts on ecosystems and human well-being. Science, Washington, v. 355, n. 6332, 2017.

PHILLIPS, SJ, ANDERSON, RP, DUDÍK, M, SCHAPIRE, RE, BLAIR, ME. Opening the black box: an open-source release of Maxent. Ecography, Hoboken, v. 40, p. 887893, 2017.

R CORE TEAM. R: A Language and Environment for Statistical Computing. R Foundation for Statistical Computing, Vienna, Austria, 2019.

RAMOS DM, CHAVES CL, BORTOLUZZI RCL, MANTOVANI A. Florística de Floresta Ombrófila Mista Altomontana e de campos em Urupema, Santa Catarina, Brasil. Revista Brasileira de Biociências, Porto Alegre, v. 9, n. 2, p. 156-166, 2011.
SANTOS CS, CHIOSSI RY, AVILA AL, GASPARIN E. Levantamento florístico e fitossociológico de um fragmento florestal no município de Faxinal dos Guedes, SC. Unoesc \& Ciência, Joaçaba, v. 3, n. 1, p. 7-22, 2012.

SAWCZUK AR. Florística e estrutura horizontal no período 2002-2008 de um fragmento de Floresta Ombrófila Mista no Centro-Sul do estado Paraná. 2009. Dissertação (Mestrado em Ciências Florestais) - UNICENTRO, Guarapuava, 2009.

SHABANI F, KUMAR L, AHMADI M. Assessing accuracy methods of species distribution models: AUC, specificity, sensitivity and the true skill statistic. GJHSS: Geography Geo-Sciences Environmental Science and Disasters Management, Framingham, v. 18, n. 91, p. 6-18, 2018.

SIQUEIRA SF, HIGUCHI P, SILVA AC. Contemporary and future potential geographic distribution of Cedrela fissilis Vell. under climate change scenarios. Revista Árvore, Viçosa, v. 43, n. 3, e430306, 2019.

SIMON MF et al. The evolutionary history of Mimosa (Leguminosae): toward a phylogeny of the sensitive plants. American Journal of Botany, Hoboken, v. 98, n. 7, p. 1201-1221, 2011.

SVENNING J, SANDEL B. Disequilibrium vegetation dynamics under future climate change. American Journal of Botany, Hoboken, v. 100, n. 7, p. 1266-1286, 2013.

SOBIERAJSKI GR. Estrutura genética em populações de bracatinga (Mimosa 
scabrella Benth.) por marcador isoenzimático e caracteres quantitativos. 2004. Dissertação (Mestrado em Recursos Florestais) - ESALQ/USP. Piracicaba, 2004.

STEENBOCK W, SIMINSKI A, FANTINI AC, REIS MS. Ocorrência da bracatinga (Mimosa scabrella Benth.) em bracatingais manejados e em florestas secundárias na região do planalto catarinense. Revista Árvore, Viçosa, v. 35, n. 4, p. 845-857, 2011.

THUILLER W, GEORGES D, ENGLER R, BREINER F. Ensemble platform for species distribution modeling. $\mathrm{R}$ package version 3.164, 2019.

TOLEDO M, POORTER L, PEÑARODRIGUES M, ALARCÓN A, BALCÁZAR J, LEAÑO C, LICONA JC, LLANQUE O, VROOMANS V, ZUIDEMA $\mathrm{P}$, BONGERS F. Climate is a stronger driver of tree and forest growth rates than soil and disturbance. Journal of Ecology, Londres, v. 99, n. 1, p. 254-264, 2011.

URBAN MC. Accelerating extinction risk from climate change. Science, Washington, v. 348, n. 6234, p. 571-573, 2015.

WEBBER BL, SCOTT JK. Rapid global change: implications for defining natives and aliens. Global Ecology and Biogeography, Hoboken, v. 21, n. 3, p. 305-311, 2012.

WILSON OJ, WALTERS RJ, MAYLE FE, LINGNER DV, VIBRANS AC. Cold spot microrefugia hold the key to survival for Brazil's Critically Endangered Araucaria tree. Global Change Biology, Hoboken, v. 25, p. 4339-4351, 2019. 